\section{Exames de confirmação}

Os exames de confirmação ou de exclusão do quadro de TEP e de TVP incluem a cintilografia pulmonar de ventilação e perfusão, angio-TC, angio-RM, a arteriografia pulmonar por cateter com subtração digital e a ultrassonografia dos membros inferiores.

A ultrassonografia dos membros inferiores com Doppler a cores é um excelente método para o diagnóstico de TVP dos membros inferiores, com acurácia de 99\% acima do joelho e de $90 \%$ abaixo do joelho. ${ }^{(1)}$ Esse método não invasivo pode ser realizado até junto ao leito de pacientes acamados e não tem contraindicações; assim, é uma excelente opção para o estudo de TVP profunda isoladamente. Outros métodos para o estudo do sistema venoso profundo estão disponíveis, tais como a veno-TC, a venorressonância magnética e flebografia convencional. Entretanto, pela praticidade e custo, a ultrassonografia é o método de escolha.

Em pacientes sintomáticos para TVP, a ultrassonografia pode ser utilizada como método inicial de investigação, uma vez que encontrada a TVP, pode-se iniciar a anticoagulação.(B)

Para o estudo de TEP, os exames podem ser divididos em métodos indiretos, pelos quais se buscam sinais que se correlacionam com a presença de tromboembolia, como a cintilografia pulmonar de ventilação e perfusão, ou exames diretos, que buscam a identificação do trombo propriamente dito, como a angio-TC, a angio-RM e a angiografia convencional.

A cintilografia pulmonar de ventilação e perfusão é um método cujo desempenho diagnóstico foi criteriosamente estudado no final dos anos 1980 pelo PIOPED $^{(2)}$ e, quando associado à probabilidade clínica para TEP, possui alto VPN quando o resultado é normal (VPN $=97 \%)$, assim como alto VPP quando o resultado é de alta probabilidade (VPP $=92-99 \%)$. No entanto, resultados de moderada probabilidade não auxiliam no processo de decisão e conduta. ${ }^{(3)}$

Apesar dos critérios cintilográficos terem sido revistos, ainda há um grande número de pacientes que tem resultado de moderada probabilidade, principalmente aqueles com radiografias alteradas ou aqueles com comorbidades cardíacas ou pulmonares, como embolias prévias e DPOC, entre outras. ${ }^{(4)}$ Uma situação que não é incomum, porém frequentemente interpretada erroneamente pelos clínicos devido a sua nomen- clatura confusa, é a ocorrência de resultados de cintilografia pulmonar com baixa probabilidade de TEP em pacientes com alta suspeita clínica. Nesses casos, a TEP pode ocorrer em até 40\%, sendo obrigatório o prosseguimento da investigação, apesar da "baixa" probabilidade. Outras situações que merecem destaque é a possibilidade de resultados falso-positivos em pacientes com asma (vasoconstrição reflexa) ou com TEP prévia (déficit perfusional persistente).

0 emprego do exame de cintilografia pulmonar de ventilação e perfusão em pacientes sem comorbidades e com radiografias normais propicia um melhor rendimento desse estudo, com menos resultados indeterminados, sendo uma excelente opção na investigação inicial das suspeitas de TEP. ${ }^{(5)} \mathrm{A}$ associação dos dados de probabilidade clínica para TEP ao exame de cintilografia apenas de perfusão (comparados à radiografia de tórax) também demonstra boa acurácia diagnóstica, com redução do custo do exame.

Um exame de cintilografia pulmonar normal é suficiente para afastar o diagnóstico de TEP. (A)

Um exame de cintilografia pulmonar com alta probabilidade para TEP em um paciente com alta probabilidade clínica é suficiente para confirmar esse diagnóstico.(A)

A utilização da TC para o estudo de TEP evoluiu muito em consequência dos avanços tecnológicos, e, no recente PIOPED $11,{ }^{(6)}$ estudou-se o papel da TC com múltiplos detectores no diagnóstico de TEP. Nesse estudo, os autores demonstraram que o exame de angioTC das artérias pulmonares, realizada com tomógrafo de múltiplos detectores, (incluindo a pesquisa de TVP dos membros inferiores realizada por TC) tem alto VPP (96\%) em pacientes com alta probabilidade clínica para TEP, assim como também alto VPN (97\%) em pacientes de baixa probabilidade clínica. Nos pacientes com probabilidade clínica intermediária, tanto o VPN quanto o VPP foram de 92\%.

A angio-TC possibilita, além do estudo angiotomográfico, a avaliação da aorta, do parênquima pulmonar, da caixa torácica e do espaço pleural, permitindo assim a realização de diagnósticos alternativos nos casos de suspeita de TEP.

Os valores preditivos e a acurácia da angioTC se assemelham bastante àqueles encontrados 
nos estudos de cintilografia, sendo, portanto, possível utilizar os mesmos graus de recomendação, principalmente se considerarmos as demais vantagens já mencionadas da angio-TC, além da maior reprodutibilidade do método. ${ }^{(7)}$

Um exame de angio-TC negativo para TEP e TVP em pacientes com baixa probabilidade clínica para TEP é suficiente para afastar esse diagnóstico.(A)

Um exame de TC positivo para TEP ou TVP em um paciente com alta probabilidade clínica é suficiente para confirmar esse diagnóstico.(A)

A angio-RM é uma alternativa à angio-TC, pois, através desse método, também se pode realizar um estudo angiográfico, assim como outras técnicas, como a perfusão pulmonar, que podem auxiliar no diagnóstico da TEP.

A angio-RM tem algumas vantagens em relação à angio-TC, sendo as principais a ausência de radiação e o meio de contraste utilizado (gadolínio), que pode ser utilizado em pacientes com alergia a contraste iodado. Pacientes com insuficiência renal, que até pouco tempo atrás eram um nicho para a ressonância magnética, atualmente têm esta indicação proibida pela ocorrência de fibrose sistêmica (incluindo alterações cutâneas e infiltração visceral) relacionada ao uso do gadolínio nesses pacientes. Além disso, o exame de angio-RM possibilita a realização de outras técnicas, como a perfusão, a quantificação de fluxo nos grandes vasos e a avaliação da função cardíaca. As principais desvantagens do método são a menor resolução espacial, maior custo, maior complexidade, menor disponibilidade e a dificuldade de monitorar pacientes graves no interior do equipamento, devido ao alto campo magnético. A principal indicação atual da ressonância magnética é como método alternativo à TC em pacientes com alergia ao contraste iodado.(C)

A angiografia pulmonar, consagrada como padrão ouro no diagnóstico de embolia, é um método invasivo, com baixa morbidade e mortalidade. ${ }^{(8)}$ Apesar de essa ser considerada o padrão ouro, vale ressaltar que esse exame também apre- senta limitações de sensibilidade e especificidade no estudo dos vasos subsegmentares. ${ }^{(9)}$ Por ser um método invasivo e também de baixa disponibilidade, seu emprego como método inicial é usualmente limitado aos pacientes instáveis com contraindicação ao uso de trombolíticos e que podem se beneficiar da trombectomia por cateter, embora não tenha sido demonstrado ainda o impacto dessa abordagem.(C). Nos pacientes estáveis, a indicação da angiografia ocorre quando os resultados dos exames não invasivos são inconclusivos, ou quando há discordância entre os mesmos e a clínica.(A) ${ }^{(5)}$

\section{Referências}

1. Mani R, Regan F, Sheridan J, Batty V. Duplex ultrasound scanning for diagnosing lower limb deep vein thrombosis. Dermatol Surg. 1995;21(4):324-6.

2. Value of the ventilation/perfusion scan in acute pulmonary embolism. Results of the prospective investigation of pulmonary embolism diagnosis (PIOPED). The PIOPED Investigators. JAMA. 1990;263(20):2753-9.

3. Miniati M, Pistolesi M, Marini C, Di Ricco G, Formichi $\mathrm{B}$, Prediletto $\mathrm{R}$, et al. Value of perfusion lung scan in the diagnosis of pulmonary embolism: results of the Prospective Investigative Study of Acute Pulmonary Embolism Diagnosis (PISA-PED). Am J Respir Crit Care Med. 1996;154(5):1387-93.

4. Jacobsson $\mathrm{H}$. Time to reconsider the PIOPED criteria? Eur J Nucl Med. 2000;27(3):368.

5. British Thoracic Society Standards of Care Committee Pulmonary Embolism Guideline Development Group. British Thoracic Society guidelines for the management of suspected acute pulmonary embolism. Thorax. 2003;58(6):470-83.

6. Stein PD, Fowler SE, Goodman LR, Gottschalk A, Hales CA, Hull RD, et al. Multidetector computed tomography for acute pulmonary embolism. N Engl J Med. 2006;354(22):2317-27.

7. Quiroz R, Kucher N, Zou KH, Kipfmueller F, Costello $\mathrm{P}$, Goldhaber SZ, et al. Clinical validity of a negative computed tomography scan in patients with suspected pulmonary embolism: a systematic review. JAMA. 2005;293(16):2012-7.

8. Wallis JW, van Beek EJ, Reekers JA, van Rooij WJ, Oudkerk M. Reserve concerning pulmonary angiography in pulmonary embolism is not justified. [Article in Dutch]. Ned Tijdschr Geneeskd. 1997;141(12):578-81.

9. Quinn MF, Lundell CJ, Klotz TA, Finck EJ, Pentecost M, McGehee WG, et al. Reliability of selective pulmonary arteriography in the diagnosis of pulmonary embolism. AJR Am J Roentgenol. 1987;149(3):469-71. 